\title{
Hepatoid Adenocarcinoma of the Anterior Skull Base: A Case Report
}

\author{
Rebecca Zener, B.Sc., ${ }^{1}$ Olga Gologan, M.D., ${ }^{2}$ Noah Sands, M.D., ${ }^{1}$ \\ and Anthony G. Zeitouni, M.D., M.Sc. ${ }^{1}$
}

We report the first case of hepatoid adenocarcinoma of the skull base, as well as the first reported case in the head and neck region. Hepatoid adenocarcinoma is a rare, aggressive, extrahepatic malignancy with a distinct morphological similarity to hepatocellular carcinoma, in the absence of primary hepatic disease. A 45-year-old man presented with sinus headaches and retro-orbital pain and was found to have a nasopharyngeal mass on endoscopy and a large, destructive sinonasal mass extending intracranially on imaging. Histo- and cytopathological features were similar to hepatocellular carcinoma, and the cells were immunoreactive for $\alpha$-fetoprotein, epithelial membrane antigen, periodic acid-Schiff, cytokeratin (CK)8/18, CK19, and S100.

KEYWORDS: Hepatoid adenocarcinoma, skull base tumors, AFP, sinonasal tumors

$\mathrm{H}_{\text {epatoid tumors are a group of malignancies }}$ with histological features resembling those of hepatocellular carcinoma (HCC). These rare tumors are most often found to arise in abdominal or pelvic organs. Gross and microscopic pathological features include neoplastic cells proliferating in a trabecular or sheetlike fashion with abundant, eosinophilic cytoplasm. A solid pattern of cellular arrangement with sinusoid-like spaces lined by endothelium is observed and shared with the HCC. ${ }^{1}$ Presence of an extrahepatic primary tumor whose morphology is highly consistent with HCC and immunohistochemistry positive for $\alpha$-fetoprotein (AFP), in the absence of a liver primary or other extrahepatic disease, is consistent with a hepatoid malignancy. ${ }^{2}$

\section{CASE REPORT}

A 45-year-old man presented to an otolaryngologist at another center with a history of "sinus headaches" and retro-orbital pain. Past medical history was significant for controlled hypertension. He was a nondrinker and had a remote history of a few pack-years of smoking as a teenager. On examination, he was found to have a nasopharyngeal mass and facial hypesthesia in the V1 and V2 distributions; however, there was no palpable cervical lymphadenopathy. Computed tomography (CT) of the head and neck revealed a T4N0, destructive left ethmoidosphenoidal mass invading the maxillary sinus, pterygopalatine fossa, infratemporal fossa, and left cavernous sinus. Magnetic resonance imaging (MRI) of the paranasal sinuses and skull base demonstrated a $5.7 \times 4.4-\mathrm{cm}$ mass compressing the left orbit and optic nerve, with invasion of the anterior and middle cranial fossa with encroachment on the medial aspect of the left temporal lobe (Fig. 1). Chest, abdomen, and pelvis CT were negative for distant metastases. Laboratory investigations revealed normal liver enzymes and liver function tests, as well as normal serum AFP of $2 \mu \mathrm{g} / \mathrm{L}$
Departments of ${ }^{1}$ Otolaryngology-Head and Neck Surgery and ${ }^{2}$ Pathology, McGill University Health Center, Montreal, Quebec, Canada.

Address for correspondence and reprint requests: Anthony G. Zeitouni, M.D., M.Sc., Royal Victoria Hospital, 687 Pine Avenue West, E4.45, Montreal, Quebec, Canada, H3A 1A1 (e-mail: anthony. zeitouni@mcgill.ca).
Skull Base Rep 2011;1:95-98. Copyright (C) 2011 by Thieme Medical Publishers, Inc., 333 Seventh Avenue, New York, NY 10001, USA. Tel: +1(212) 584-4662.

Received: December 14, 2010. Accepted: December 21, 2010. Published online: April 11, 2011.

DOI: http://dx.doi.org/10.1055/s-0031-1276723.

ISSN 2157-6971. 
A
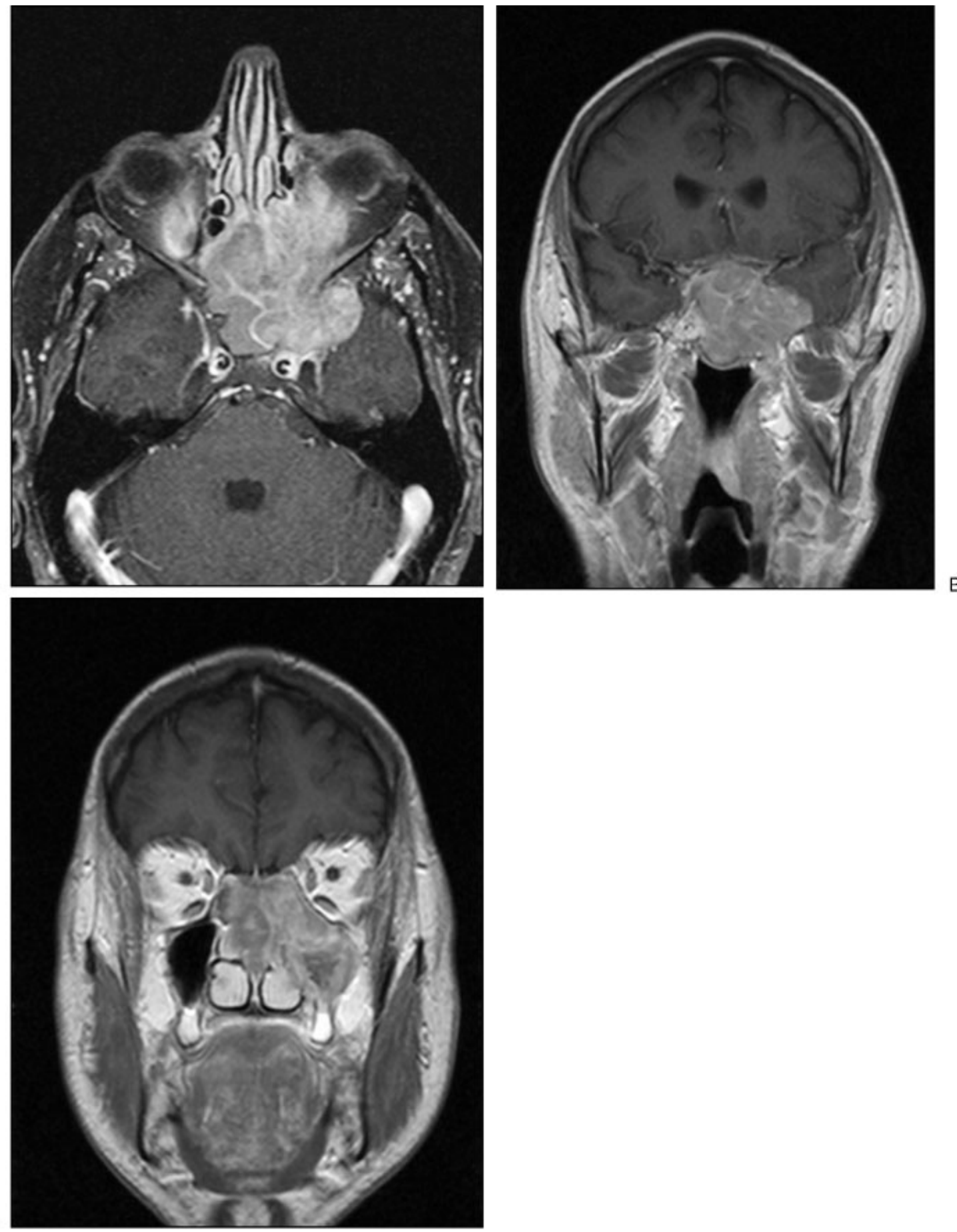

Figure 1 Prechemoradiotherapy T1-weighted fat-saturated magnetic resonance images: axial (A) and coronal (B, C).

(normal range $=0$ to 9 ) and normal carcinoembryonic antigen (CEA) of $1 \mu \mathrm{g} / \mathrm{L}$ (normal range $=0$ to 4.9 ).

Biopsy of the nasopharyngeal mass was performed; the initial diagnosis was squamous cell carcinoma, and the patient was referred to our center for second opinion and a radiation oncology consultation. At our center, an extensive immunopanel was performed on the specimen and reviewed by two pathologists.
The histological slides demonstrated a solid trabecular proliferation of cells, supported by a delicate capillary vascular frame, invading fibroconnective tissue and bone. The neoplastic cells were medium to large and relatively monotonous, with medium to abundant, clear and vacuolated pale eosinophilic cytoplasm. The nuclei were round and reveal open, lacey chromatin with conspicuous eosinophilic nucleoli 
A
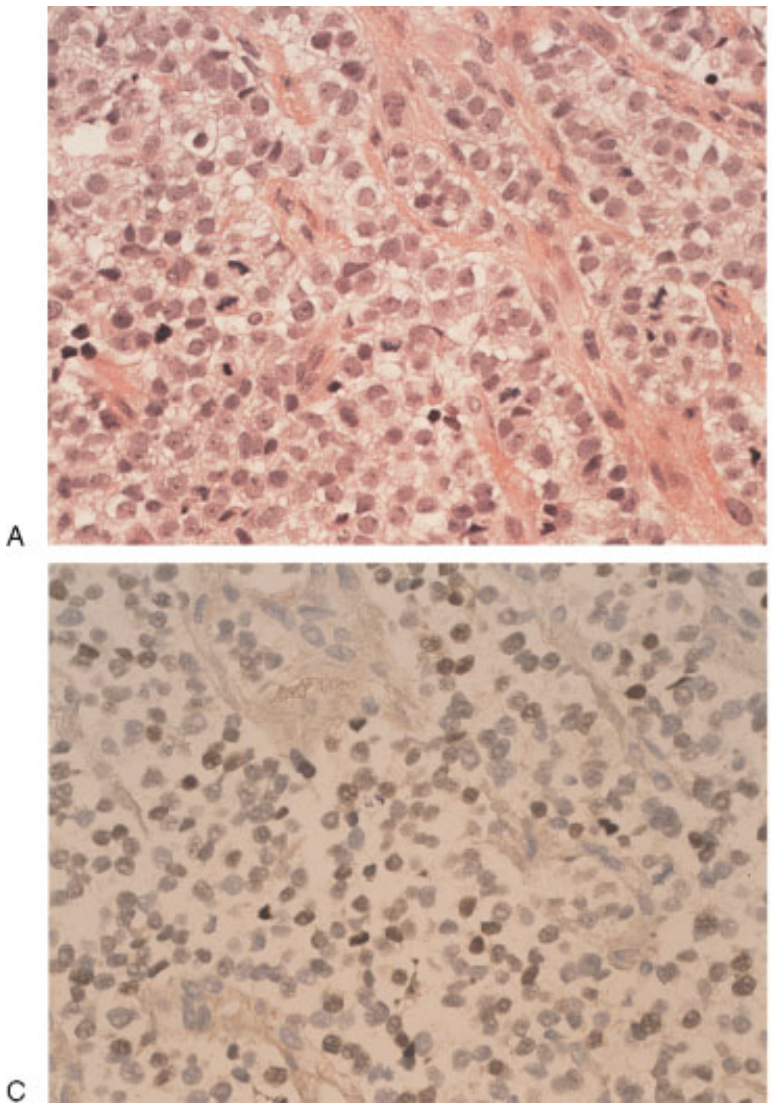

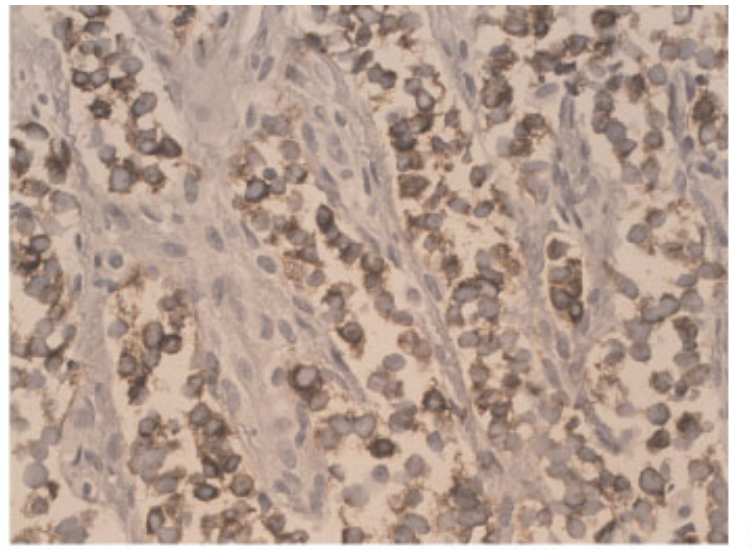

B

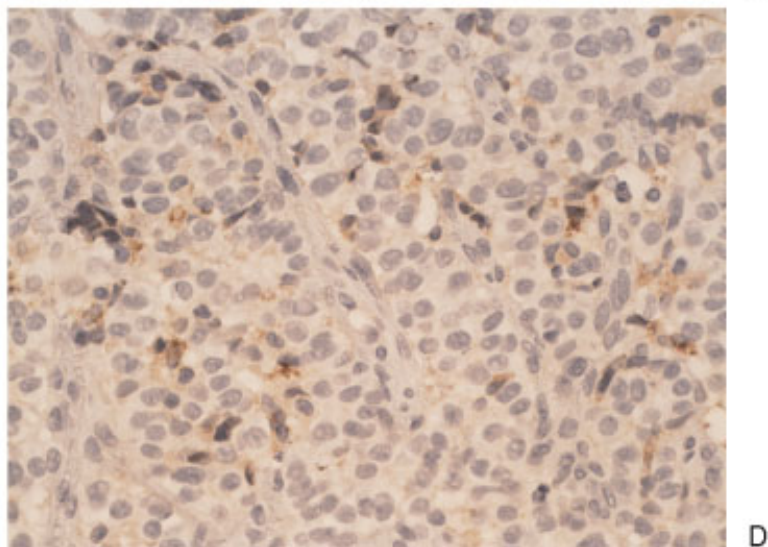

Figure 2 (A) Histological appearance. The tumor is composed of medium to large, monotonous cells with abundant clear and vacuolated pale eosinophilic cytoplasm, with round nuclei containing open lacey chromatin (hematoxylin and eosin staining, original magnification $400 \times$ ). (B) The tumor stains diffusely for cytokeratin 8/18/19 (immunohistochemistry, original magnification $400 \times$ ). (C) The tumor stains focally for S100 (immunohistochemistry, original magnification $400 \times$ ). (D) The tumor stains focally for $\alpha$-fetoprotein (immunohistochemistry, original magnification $400 \times$ ).

(Fig. 2A). Frequent mitotic figures were present, with an absence of necrosis. On immunohistochemistry, the tumor stained positive for epithelial membrane antigen and periodic acid-Schiff (glycogen), cytokeratin (CK)8/18/19 (Fig. 2B), S100 (Fig. 2C), and AFP (Fig. 2D), focally.

Stains for CK5/6 and p65 (squamous cell markers); CK7, CK20, thyroid transcription factor-1, and CDX-2 (lung and gastrointestinal tract markers); smooth muscle actin and calponin (myoepithelial markers); GCDFP-15 (breast marker); prostate-specific antigen (prostate marker); CD10, CD30, RCC (renal cell carcinoma markers); hepatocyte and A1AT (HCC markers); inhibin (adrenal cortical marker); synaptophysin, chromogranin, (neuroendocrine markers); CEA (mono- and polyclonal), placental alkaline phosphatase (germ cell marker), vimentin, follicule-stimulating hormone, luetinizing hormone, adrenocorticotropic hormone, Melan-A, CD56, and Alcian blue $\mathrm{pH} 2.5$ were negative.

The histo- and cytomorphological features are very similar to $\mathrm{HCC}$ and a final diagnosis of undifferentiated adenocarcinoma with hepatoid morphology was made.
The case was reviewed at our multidisciplinary tumor board, and the patient underwent induction chemotherapy with docetaxel/cisplatin/5-fluorouracil, followed by radiotherapy, 70 Gy in 35 fractions. Follow-up MRI of the paranasal sinuses and skull base revealed greatly diminished tumor bulk with persistence of disease in the sphenoethmoidal sinuses, along the skull base and left middle cranial fossa, with diminished enhancement of the orbits (Fig. 3). The patient received sorafenib maintenance therapy, and follow-up positron emission tomography revealed no evidence of metabolically active neoplastic disease.

\section{DISCUSSION}

This is the first reported case of a primary skull base/ nasopharyngeal hepatoid adenocarcinoma (HAC). Hepatoid differentiation is a feature that has been described previously in yolk sac tumors of the skull base; however, it has been absent in descriptions of non-germ-cell origin of the skull base and the head and neck. Although two of the most common locations of HAC include the stomach $^{3-5}$ and ovary, ${ }^{6,7}$ multiple reports of tumors of non-germ-cell origin have been reported in the colon, 


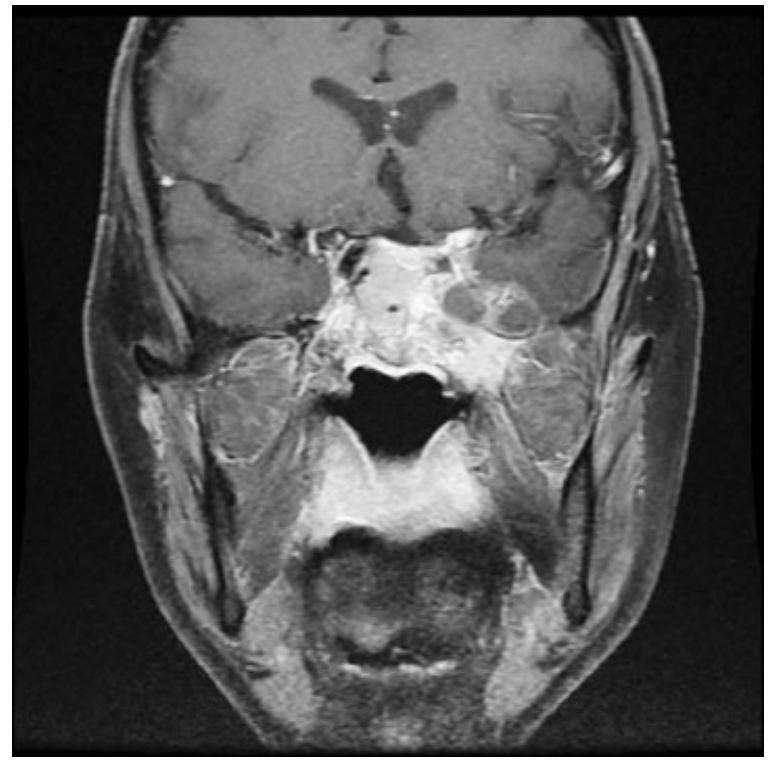

Figure 3 Postchemoradiotherapy, prior to sorafenib maintenance therapy; postgadolinium T1-weighted magnetic resonance coronal image. The large tumor has decreased in size and demonstrates extensive, coarse calcifications.

duodenum, gallbladder, ${ }^{8}$ kidney, lower esophagus, lung, ${ }^{9}$ pancreas, ${ }^{10,11}$ rectum, urinary bladder,${ }^{12}$ uterine cervix, ${ }^{13}$ and uterus. ${ }^{14}$ Additionally, review of the literature revealed one case of HAC localized to shoulder soft tissue. ${ }^{15}$ The majority of HAC occur in middle-aged to elderly individuals. ${ }^{10}$

Microscopic descriptions of HAC in other sites such as the lung, pancreas, and gallbladder have included a heterogeneous tubular proliferative pattern with large neoplastic cells containing abundant, eosinophilic cytoplasm with centrally positioned nuclei and an overall monotonous appearance analogous to HCC. ${ }^{1,8,9}$ The features in our case are congruent with these descriptions.

$\mathrm{CT}$ features of HAC in other anatomic locations have included heterogeneously appearing tumors with a density similar to liver tissue, demonstrating moderate enhancement post-intravenous iodine contrast. ${ }^{16}$ In our case, the large tumor had extensively eroded surrounding bone and significantly infiltrated the left orbit, cavernous sinus, sella turcica, and anterior and middle cranial fossae. This aggressive behavior is consistent with $\mathrm{HAC}$, a rare extrahepatic neoplasm, known to invade adjacent tissue. ${ }^{14}$

Our patient's serum AFP was normal. Although the majority of HACs produce AFP, up to one-third have a normal serum level. ${ }^{15}$ Additionally, it has been suggested a diagnosis of HAC should depend on the histopathologic features rather than AFP production, as HAC has been shown to display similar biological behavior regardless of AFP production. ${ }^{5}$

Extensive clinical, biochemical, and radiological metastatic workup excluded another possible primary origin. Moreover, the aforementioned staining pattern refuted distant metastases from squamous, lung, gastro- intestinal, hepatic, renal, pancreatic, germ cell, adrenal, neuroendocrine, or salivary gland origin. Accordingly, having excluded other possible primary tumors and given the histo- and cytomorphological resemblance between our patient's nasopharyngeal/skull base tumor and $\mathrm{HCC}$, the case was classified appropriately as a HAC arising from the skull base.

\section{REFERENCES}

1. Ishikura H, Kanda M, Ito M, Nosaka K, Mizuno K. Hepatoid adenocarcinoma: a distinctive histological subtype of alpha-fetoprotein-producing lung carcinoma. Virchows Arch A Pathol Anat Histopathol 1990;417:73-80

2. Terracciano LM, Glatz K, Mhawech P, et al. Hepatoid adenocarcinoma with liver metastasis mimicking hepatocellular carcinoma: an immunohistochemical and molecular study of eight cases. Am J Surg Pathol 2003;27:1302-1312

3. Lu CC, De-Chuan C, Lee HS, Chu HC. Pure hepatoid adenocarcinoma of the stomach with spleen and lymph-node metastases. Am J Surg 2010;199:e42-e44

4. Ishikura H, Fukasawa Y, Ogasawara K, Natori T, Tsukada Y, Aizawa M. An AFP-producing gastric carcinoma with features of hepatic differentiation. A case report. Cancer 1985;56: 840-848

5. Nagai E, Ueyama T, Yao T, Tsuneyoshi M. Hepatoid adenocarcinoma of the stomach. A clinicopathologic and immunohistochemical analysis. Cancer 1993;72:1827-1835

6. Ishikura H, Scully RE. Hepatoid carcinoma of the ovary. A newly described tumor. Cancer 1987;60:2775-2784

7. Yiğit S, Uyaroğlu MA, Ku Z, Ekinci N, Oztekin O. Hepatoid carcinoma of the ovary: immunohistochemical finding of one case and literature review. Int J Gynecol Cancer 2006;16:14391441

8. Nakashima H, Nagafuchi K, Satoh $H$, et al. Hepatoid adenocarcinoma of the gallbladder. J Hepatobiliary Pancreat Surg 2000;7:226-230

9. Hayashi Y, Takanashi Y, Ohsawa H, Ishii H, Nakatani Y. Hepatoid adenocarcinoma in the lung. Lung Cancer 2002; 38:211-214

10. Hameed O, Xu H, Saddeghi S, Maluf H. Hepatoid carcinoma of the pancreas: a case report and literature review of a heterogeneous group of tumors. Am J Surg Pathol 2007;31: 146-152

11. Paner GP, Thompson KS, Reyes CV. Hepatoid carcinoma of the pancreas. Cancer 2000;88:1582-1589

12. Burgués O, Ferrer J, Navarro S, Ramos D, Botella E, Llombart-Bosch A. Hepatoid adenocarcinoma of the urinary bladder. An unusual neoplasm. Virchows Arch 1999;435:71-75

13. Kato K, Suzuka K, Osaki T, Itami M, Tanaka N. Primary hepatoid adenocarcinoma of the uterine cervix. Int J Gynecol Cancer 2007;17:1150-1154

14. Gopaldas R, Kunasani R, Plymyer MR, Bloch RS. Hepatoid malignancy of unknown origin-a diagnostic conundrum: review of literature and case report of collision with adenocarcinoma. Surg Oncol 2005;14:11-25

15. Fornasa F. Soft-tissue localization of hepatoid adenocarcinoma: first case report. Case Rep Oncol 2010;3:212-217

16. Wu Z, Upadhyaya M, Zhu H, Qiao Z, Chen K, Miao F. Hepatoid adenocarcinoma: computed tomographic imaging findings with histopathologic correlation in 6 cases. J Comput Assist Tomogr 2007;31:846-852 